\title{
Genetic studies of African populations: an overview on disease susceptibility and response to vaccines and therapeutics
}

\author{
Giorgio Sirugo • Branwen J. Hennig • Adebowale A. Adeyemo • Alice Matimba • Melanie J. Newport • \\ Muntaser E. Ibrahim • Kelli K. Ryckman - Alessandra Tacconelli • Renato Mariani-Costantini · \\ Giuseppe Novelli $\cdot$ Himla Soodyall $\cdot$ Charles N. Rotimi $\cdot$ Raj S. Ramesar $\cdot$ Sarah A. Tishkoff $\cdot$ Scott M. Williams
}

Published online: 1 August 2008

(C) Springer-Verlag 2008

\section{Erratum to: Hum Genet (2008) 123:557-598 DOI 10.1007/s00439-008-0511-y}

The authors forgot to include the following crucial footnote to Tables 1,2 and 3:

A regular updating of the tables reporting genetic associations of susceptibility to malaria, tuberculosis and HIV/ AIDS will be posted on the web site of the Center for

The online version of the original article can be found under doi: 10.1007/s00439-008-0511-y.

\section{G. Sirugo ( $\square)$}

Medical Research Council Laboratories, Fajara,

The Gambia, West Africa

e-mail: sirugo.giorgio@fbfrm.it; gsirugo@gmail.com

\section{G. Sirugo · A. Tacconelli · G. Novelli}

Medical Genetics Unit, Ospedale S. Pietro FBF, Rome, Italy

G. Sirugo · A. Tacconelli · G. Novelli

Department of Biopathology and Diagnostic Imaging,

School of Medicine, Tor Vergata University, Rome, Italy

\section{B. J. Hennig}

London School of Hygiene and Tropical Medicine, London, UK

A. A. Adeyemo - C. N. Rotimi

National Human Genome Center, Howard University,

College of Medicine, Washington, DC, USA

\section{A. Matimba}

Molecular Science Unit, (AiBST), Harare, Zimbabwe

M. J. Newport

Department of Medicine, Brighton and Sussex Medical School,

University of Sussex, Brighton, UK
Human Genetics Research at Vanderbilt University at the following address: http://chgr.mc.vanderbilt.edu/page/ supplementary-data.

\section{E. Ibrahim}

Department of Molecular Biology, Institute of Endemic Diseases,

University of Khartoum, Khartoum, Sudan

K. K. Ryckman · S. M. Williams ( $₫)$

Center for Human Genetic Research, Vanderbilt University

School of Medicine, Nashville, TN, USA

e-mail: smwilliams@chgr.mc.vanderbilt.edu

R. Mariani-Costantini

Department of Oncology and Neuroscience and Ageing Research

Center, University Gabriele d'Annunzio, Chieti, Italy

H. Soodyall

Human Genomic Diversity and Disease Research Unit,

University of the Witwatersrand, Johannesburg, South Africa

R. S. Ramesar

Division of Human Genetics,

Institute of Infectious Disease and Molecular Medicine,

University of Cape Town, Cape Town, South Africa

\section{S. A. Tishkoff}

Department of Genetics, University of Pennsylvania,

School of Medicine, Philadelphia, USA 